\title{
Intersublevel transitions in InAs/GaAs quantum dots infrared photodetectors
}

\author{
S. Maimon, E. Finkman, ${ }^{\text {a) }}$ and G. Bahir \\ Department of Electrical Engineering and Solid State Institute, Technion, Haifa 32000, Israel
}

S. E. Schacham

Department of Electrical and Electronic Engineering, College of Judea and Samaria, Ariel 44837, Israel

J. M. Garcia and P. M. Petroff

Center for Quantized Electronic Structures (QUEST) and Materials Department, University of California, Santa Barbara, California 93106

(Received 3 February 1998; accepted for publication 31 July 1998)

Thermal generation rate in quantum dots (QD) can be significantly smaller than in quantum wells, rendering a much improved signal to noise ratio. QDs infrared photodetectors were implemented, composed of ten layers of self-assembled InAs dots grown on GaAs substrate. Low temperature spectral response shows two peaks at low bias, and three at a high one, polarized differently. The electronic level structure is determined, based on polarization, bias, and temperature dependence of the transitions. Although absorbance was not observed, a photoconductive signal was recorded. This may be attributed to a large photoconductive gain due to a relatively long lifetime, which indicates, in turn, a reduced generation rate. (C) 1998 American Institute of Physics.

[S0003-6951(98)02140-8]

The research of quantum dots (QDs) was greatly advanced by the rediscovering of the Stranski-Krastanov ${ }^{1}$ growth method. Using this relatively simple method, selfassembled QDs with a narrow size variance, and a high surface coverage efficiency are grown using molecular beam epitaxy, making use of the strain between lattice mismatched semiconductors. $^{2-5}$

Several theoretical models of the electronic structure in QDs have been proposed ${ }^{6-9}$ to account for the observed intersublevel energies. ${ }^{10-12}$ The agreement between theory and experiments still remains controversial. Indeed, the shape and sizes of the QDs which strongly affect the electronic levels, are not accurately determined. ${ }^{13}$

Intersublevel transitions in the conduction band of QDs were first studied using a combination of capacitance and far-infrared (IR) spectroscopy, ${ }^{10-12}$ transitions in both conduction and valence bands were observed. Mid IR photoconductivity was observed in self organized InAs/ $\mathrm{Al}_{1-x} \mathrm{Ga}_{x} \mathrm{As}$ clusters. ${ }^{14}$ Its interpretation, however, was difficult due to the complexity of the sample. The studies of both conduction and valence intersublevel transitions using photoinduced IR spectroscopy on both undoped and doped samples has greatly advanced the knowledge on the subject. ${ }^{15-17}$ We have recently reported preliminary results of implementing quantum dot infrared photodetector. ${ }^{18}$ In this letter we report the investigation of intersublevel transitions in QDIPs. The photoconductive spectral response is analyzed, along with its polarization, voltage dependence, and temperature dependence.

The structure investigated in this study is composed of ten layers of self assembled, InAs dots grown on a GaAs substrate. The InAs QDs layers were separated by $10 \mathrm{~nm}$ GaAs barriers. These barriers were delta $n$ doped in their

${ }^{a)}$ Electronic mail: finkman@ee.technion.ac.il center by $\mathrm{Si}$ to a nominal donor concentration of 1-2 $\times 10^{11} \mathrm{~cm}^{-2}$. The dot density is between 2 and 4 $\times 10^{10} \mathrm{~cm}^{-2}$. Because of the small spacing between the layers the QDs are stacked on top of each other along the growth direction while their in-plane positions are random. The concentration of dots per layer is constant, and the periodicity of the structure along the growth direction is excellent $(+/-$ one monolayer). These layers were sandwiched between top and bottom AlAs/GaAs undoped superlattices. The dots are found to be lens shaped, with an average diameter of $20 \mathrm{~nm}$, and an average height of about $3 \mathrm{~nm} .{ }^{19}$ These dots can contain up to two electrons in their $s$ shell and four in their $p$ shell. The doping concentration was set such that the free electrons will partially fill the dots, without spilling over to the barriers, to avoid vertical conduction by free electrons in the barrier layers.

Attempts to measure infrared absorption were made using a $45^{\circ}$ wedge. The configuration of the incoming IR beam is set either in the $s$ (electric field parallel to the layer plane) or $p$ polarization (perpendicular to the layer plane). Transitions polarized both in the layer plane and perpendicular to it may be observed in the latter configuration (as 50\% of the component of the electric field is along the growth axis). No absorption was obtained from the ratio between the $s$ - and the $p$-polarized signals.

Two types of detectors were fabricated. One in a conventional quantum well infrared photodetector (QWIP) configuration, i.e., a mesa structure with vertical contacts, in the $z$ direction, on bottom and top of the structure (inset in Fig. 1). The mesas were $200 \times 200 \mu \mathrm{m}^{2}$ with alloyed AuGe/ $\mathrm{Ni} / \mathrm{Au}$ contacts. Typical detector resistances were $5 \mathrm{k} \Omega$ at room temperature and increased to $200 \mathrm{k} \Omega$ at $13 \mathrm{~K}$, quite lower than the anticipated resistance for the nominal doping. The high conductivity indicates either a much higher population of the dots than designed, or electron tunneling due to coupling of the dot layers through the narrow barriers. Lat- 


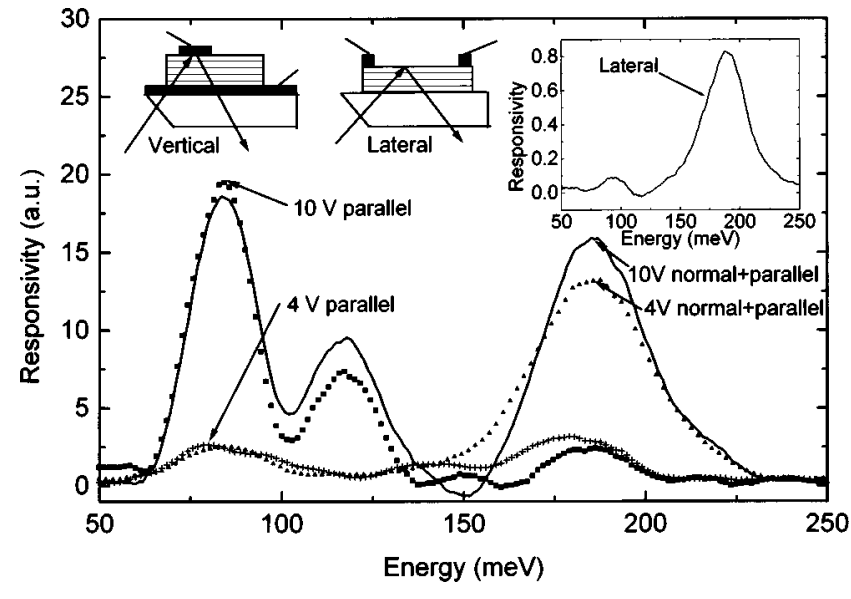

FIG. 1. QDIP responsivity as a function of photon energy at parallel polarization (dotted line) and at $45^{\circ}$ (full line). Measurement taken at $13 \mathrm{~K}$ with 4 and $10 \mathrm{~V}$ biases. Two insets on upper left show device geometries. Inset on the right—responsivity of a lateral QDIP.

eral devices were also implemented using two contact on the mesa top (Fig. 1) to investigate the possibility of observing photoconductive signal with transport in the $x y$ plain. It should be emphasized that lateral detector structures cannot function in QWIPs, since the highly populated well layers shorten the contacts. In QDs the electrons are confined in all three dimensional (3D) therefore a lateral detector can be implemented. The $50 \times 1000 \mu \mathrm{m}^{2}$ mesas were etched to the semi-insulator substrate. Typical resistance of the lateral QDIPs was $1.5 \mathrm{k} \Omega$ at $13 \mathrm{~K}$.

Photoconductive spectra were readily obtained using a Mattson Cygnus 25 Fourier-transform infrared (FTIR). Spectra taken for the vertical detector at $13 \mathrm{~K}$, with bias of 4 and $10 \mathrm{~V}$, are presented in Fig. 1, for both polarizations. For the $4 \mathrm{~V}$ bias, two distinct lines are observed. One peaked at 85 $\mathrm{meV}$ with full width at half maximum $(\mathrm{FWHM})=26 \mathrm{meV}$, the other at $185 \mathrm{meV}$ with FWHM=37 meV. The $185 \mathrm{meV}$ transition is almost completely polarized along the growth direction (the allowed transition in QWIPs), and the $85 \mathrm{meV}$ peak is observed in both $s$ and $p$ polarizations, indicating that this line is polarized mainly parallel to the layers. The peak energies are almost independent of applied bias. For the $10 \mathrm{~V}$ bias a third line is observed at $120 \mathrm{meV}$ with $F W H M=25 \mathrm{meV}$ polarized again parallel to the layers. The intensities of the 85 and the $120 \mathrm{meV}$ peaks increase gradually with bias. An inset in Fig. 1 shows the responsivity of a lateral device, indicating the same transition energies. Note that both normal and parallel polarizations are observed in this configuration.

Temperature dependence of the spectra for the vertical device with a $4 \mathrm{~V}$ bias is shown in Fig. 2. With increased temperature, both lines shift to higher energies, the intensity of the low energy line decreases, along with a decrease of the high energy linewidth. There was no significant change in the maximum intensity of the $185 \mathrm{meV}$ peak up to $65 \mathrm{~K}$, from there on it decreases rapidly. This decrease is attributed to artifacts associated with the fast drop in the sample resistance, and a saturation of the amplifier.

The optical absorption coefficient for the transition from level $i$ to $f$ in a QD is proportional to the integral:

$\alpha \propto\left|\left\langle\psi_{i}|\mathbf{p}| \psi_{f}\right\rangle\right|^{2}$

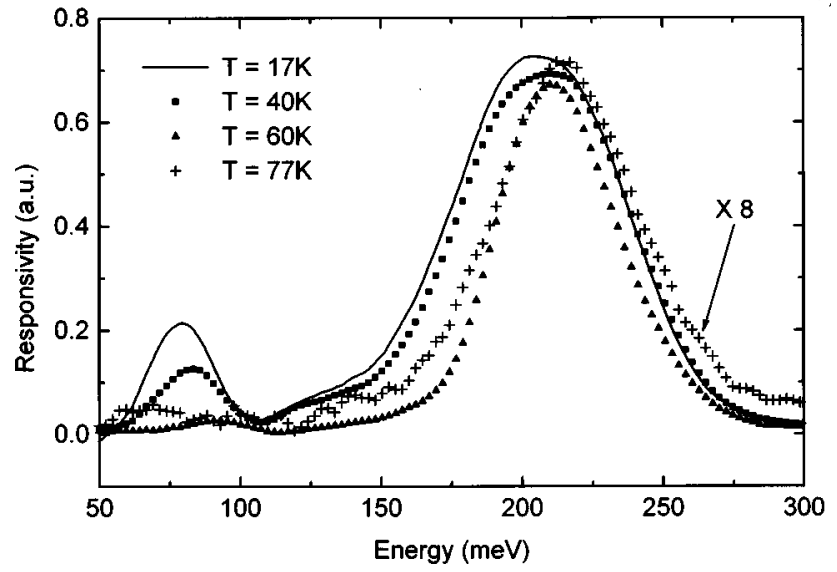

FIG. 2. QDIP responsivity as a function of temperature. Spectra are unpolarized. Measurement taken with a bias of $4 \mathrm{~V}$. The spectrum at $77 \mathrm{~K}$ is multiplied by eight.

where $p$ is the momentum operator for the corresponding transition. Conceptually, treating the QD as a box with infinite confinement potentials, with a thickness $L_{z}=L_{\perp}$ and lateral dimensions of $L_{x}=L_{y}=L_{\|}$, the wave functions in the integral are three dimensional:

$$
\psi_{\mathbf{n}}=\psi_{n x} \psi_{n y} \psi_{n z} \propto \sin \left(\frac{\pi n_{x} x}{L_{\|}}\right) \sin \left(\frac{\pi n_{y} y}{L_{\|}}\right) \sin \left(\frac{\pi n_{z} z}{L_{\perp}}\right) .
$$

The levels are represented schematically in Fig. 3 (the energy scale in this figure is arbitrary). The ground QD level has the quantum numbers $n_{x}, n_{y}, n_{z}=1,1,1$ and can contain two electrons. The second level is $1,2,1$ or $2,1,1\left(L_{x}=L_{y}\right)$ with a degeneracy of 4 , and so on. The integral defining the transitions between levels has the form:

$$
\iiint \psi_{i x} \psi_{i y} \psi_{i z} \frac{\partial}{\partial x_{k}} \psi_{f x} \psi_{f y} \psi_{f z} d x d y d z
$$

where $x_{k}=x, y, z$. Polarization selection rules will be determined by the nonzero terms of this integral. Only transitions

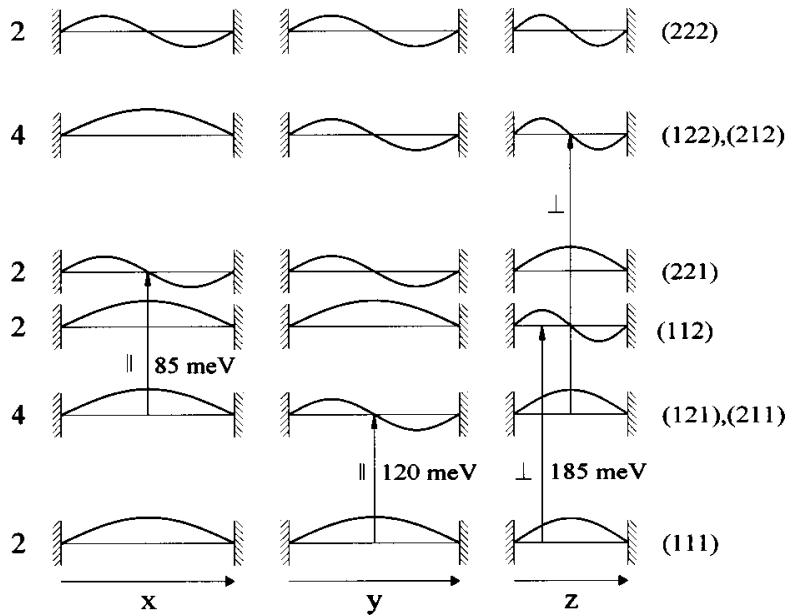

FIG. 3. Schematic representation of the wave functions for the first energy levels in a quantum dot. Arrows represent the only allowed transitions from the first and second levels. The allowed polarization is also indicated. The notations at the right correspond to the associated quantum numbers $\left(n_{x}, n_{y}, n_{z}\right)$. The numbers at the left hand of the figure denote the level degeneracy. The transitions observed in the experiment are denoted by their approximate energies. Energy values and the spacing are not to scale and are arbitrary. 
with $\Delta n=1$, from the first and second levels, are considered here, as these are by far the dominant ones. ${ }^{20}$ The presence of the derivative in the integrand implies that the allowed transitions are between energy levels in which $\Delta n=1$ only in one direction. These allowed transitions are those marked with arrows in Fig. 3. Those designated with $\|$ are allowed with in-plane polarization, and those with $\perp$ are allowed with polarization along the growth axis.

As stated before, the geometry of our dots allows a maximum of six electrons to occupy each. The chosen doping concentration was designed to fully populate the lower dot level, and partially populate the second one. Only transitions from these levels are expected to be observed. We tentatively assigned three of the transitions in Fig. 3 with the appropriate peak energies of Fig. 1. This designation is shown in Fig. 3. The polarizations of the peaks support this interpretation. The increase of the 85 and $120 \mathrm{meV}$ peaks with increasing bias indicates that for both transitions, the escape of electrons from the dot upper level to the continuum is aided by tunneling. Increasing the field narrows the tunneling barrier thus increasing the responsivity. A smaller emission probability of the $120 \mathrm{meV}$ out of the dot region may be the reason for its appearance only at higher bias. It should be mentioned, however, that the results of earlier capacitance-voltage $(C-V)$ and IR absorption studies ${ }^{6-8}$ on samples similar to ours indicated transition energies which differ from the present ones. This is not surprising since in the $C-V$ and IR measurements, there was no electronic or strain coupling between QDs since only a single QD layer was present in the structures. In fact, Coulomb interactions between coupled QDs layers have recently been shown to play an important role in the charging dynamics of QDs.

The variations with temperature (Fig. 2) are interpreted by the dots being depleted from their electrons to the barriers, with the spread of the Fermi distribution function, and the lowering of Fermi energy with increasing the temperature. The upper second level is emptied first, and the transition from it is diminished gradually. The size distribution of the dots contributes to the linewidth. As the temperature is raised and electrons are excited out of the dots, it is expected that electrons from smaller dots, i.e., with shallower energy levels, will be emptied first. As a result, only deeper dots contribute to the signal at higher temperatures and the low energy tail of the responsivity peak is reduced. The results of Schmidt $e t a l .{ }^{8}$ also indicate an upward shift of the confined levels by about $20 \mathrm{meV}$ due to the Coulomb blockade effect when the dots are filled with electrons. Here the opposite phenomenon is observed-emptying part of the dots decreases the Coulomb effect and the transitions shift to higher energies as the confined dot levels shift deeper in energies.

The bias and the temperature dependence of the peaks were measured. The spectra response is presented in arbitrary units. The magnitude of the photoconductive signal is proportional to the product of the absorption coefficient and the optical gain. The striking difference between measurement of the photoconductive signal, which was easily attained, and the absence of absorption signal, indicates a relatively large gain. This may be compared to the situation in QWIPs in which there is usually no photosignal where the absorption is absent. The most plausible interpretation is that the gain, which is proportional to the ratio of the lifetime to the transit time, is much larger in QDIP than in QWIP, indicating a large lifetime in QDIPs.

The authors would like to thank D. Schoenmann for devoted technical assistance. This research was partially supported by the Israeli Ministry of Science and Technology. Two of the authors (P.P. and J.G.) received financial support from QUEST, an NSF Science and Technology Center (DMR 11-20007).

${ }^{1}$ I. N. Stranski and L. Krastanow, Sitzungsber. Akad. Wiss. Wien Math. Naturwiss. K1 Abt. 2B Chemie 146, 797 (1937).

${ }^{2}$ M. Tabuchi, S. Noda, and A. Sasaki, Science \& Technology of Mesoscopic Structures, edited by S. Namba, C. Hamaguchi, and T. Ando (Springer, Tokyo, 1992), pp. 379-384.

${ }^{3}$ D. Leonard, M. Krishnamurthy, C. M. Reaves, S. P. Denbaars, and P. M. Petroff, Appl. Phys. Lett. 63, 3203 (1993); S. Fafard, R. Leon, D. Leonard, J. L. Merz, and P. M. Petroff, Phys. Rev. B 50, 8086 (1994).

${ }^{4}$ J.-Y. Marzin, J.-M. Gérard, A. Izrä̈l, D. Barrier, and G. Bastard, Phys. Rev. Lett. 73, 716 (1994).

${ }^{5}$ M. Grundmann, J. Christen, N. N. Ledentsov, J. Böhrer, D. Bimberg, S. S. Ruvimov, P. Werner, U. Richter, U. Gösele, J. Heydenreich, V. M. Ustinov, A. Yu. Egorov, A. E. Zhukov, P. S. Kop'ev, and Zh. I. Alferov, Phys. Rev. Lett. 74, 4043 (1995).

${ }^{6}$ P. Hawrylak, A. Wojs, D. J. Lockwood, and P. D. Wang, Surf. Sci. 361, 774 (1996).

${ }^{7}$ M. Grundmann, O. Stier, and D. Bimberg, Phys. Rev. B 52, 969 (1995).

${ }^{8}$ A. Franceschetti and A. Zunger, Appl. Phys. Lett. 68, 3455 (1996).

${ }^{9}$ L. R. C. Fonseca, J. L. Jimenez, J. P. Leburton, and R. M. Martin, Phys. Rev. B 57, 4017 (1998).

${ }^{10}$ H. D. Drexler, D. Leonard, W. Hansen, J. P. Kotthaus, and P. M. Petroff, Phys. Rev. Lett. 73, 2252 (1994); M. Fricke, A. Lorke, J. P. Kotthaus, G. Medeiros-Ribeiro, and P. M. Petroff, Europhys. Lett. 36, 197 (1996).

${ }^{11}$ G. Medeiros-Ribeiro, D. Leonard, and P. M. Petroff, Appl. Phys. Lett. 66, 1767 (1995)

${ }^{12}$ K. H. Schmidt, G. Medeiros-Ribeiro, M. Oestreich, P. M. Petroff, and G. H. Döhler, Phys. Rev. B 54, 11346 (1996).

${ }^{13}$ J. M. Garcia, G. Medeiros-Ribeiro, K. Schmidt, T. Ngo, and P. M. Petroff, Appl. Phys. Lett. 71, 2014 (1997).

${ }^{14}$ K. W. Berryman, S. A. Lyon, and M. Segev, Appl. Phys. Lett. 70, 1861 (1997).

${ }^{15}$ S. Sauvage, P. Boucaud, F. H. Julien, J. M. Gérard, and J. Y. Marzin, Appl. Phys. Lett. 82, 3396 (1997).

${ }^{16}$ S. Sauvage, P. Boucaud, F. H. Julien, J. M. Gérard, and V. Thierry-Mieg, Appl. Phys. Lett. 71, 2785 (1997).

${ }^{17}$ P. Boucaud, S. Sauvage, F. H. Julien, J. M. Gérard, and V. Thierry-Mieg, Intersubband Transitions in Quantum Wells: Physics and Devices, edited by S. S. Li and Y.-K. Su (Kluwer-Academic, Boston, 1998).

${ }^{18}$ S. Maimon, E. Finkman, G. Bahir, S. E. Schacham, and P. M. Petroff, Intersubband Transitions in Quantum Wells: Physics and Devices, edited by S. S. Li and Y.-K. Su (Kluwer-Academic, Boston, 1998).

${ }^{19}$ S. Raymond, S. Fafard, P. J. Poole, A. Wojs, P. Hawrylak, S. Charbonneau, D. Leonard, R. Leon, P. M. Petroff, and J. L. Merz, Phys. Rev. B 54, 11548 (1996).

${ }^{20}$ L. C. West and S. J. Eglash, Appl. Phys. Lett. 46, 1156 (1985). 